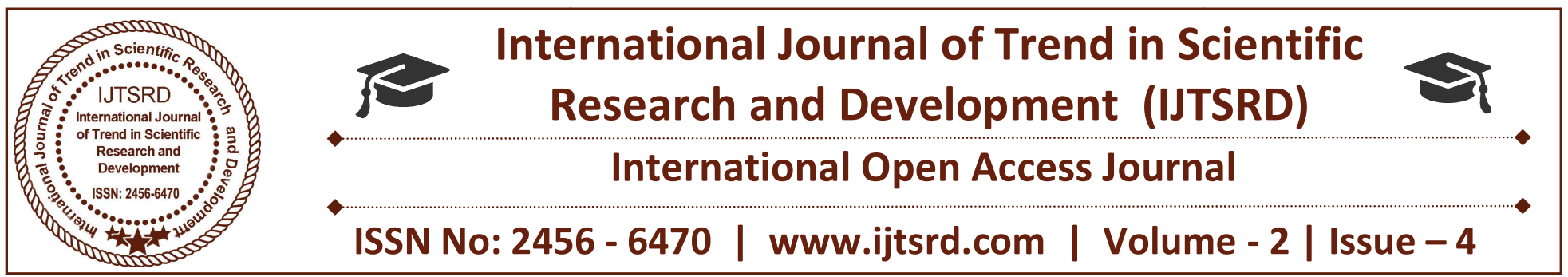

\title{
Review Paper on Wireless Sensor Networks (WSN) for Fuzzy Logy Algorithms
}

\author{
Ashish Verma ${ }^{1}$, Mukesh Patidar ${ }^{2}$ \\ ${ }^{1} \mathrm{M}$. Tech (Student), ${ }^{2}$ Professor \\ Department of Electronics and Communication Engineering, Lakshmi Narain College of Technology, \\ Indore, Madhya Pradesh, India
}

\begin{abstract}
Wireless Sensor Networks is a group of spatially dispersed dedicated sensors to monitor and record an environment's physical conditions and to organize collected data at a central location (Xiao 2004). Wireless Sensor networks (WSNs) have become one of the most interesting areas of research in the past few years. A WSN is composed of a number of wireless sensor nodes which form a sensor field and a sink. These large numbers of nodes, having the abilities to sense their surroundings, perform limited computation and communicate wirelessly form the WSNs. It is enjoy great benefits due to their very low cost, small scale, smart sensor nodes etc. Applications of wireless sensor networks such as: span environmental, animal monitoring, industrial monitoring, agricultural monitoring, automation, health monitoring and many other areas. In this paper review on WSN, literature and analysis of fuzzy loge algorithm for consider different numbers of Node and cluster like 100, 200 and 300.
\end{abstract}

Keywords: WSN, Cluster, Fuzzy logy, Sensor

\section{INTRODUCTION}

Wireless sensor network (WSN) is an ad-hoc network technology comprising even thousands of autonomic and self-organizing nodes that combine environmental sensing, data processing, and wireless networking. The applications for sensor networks range from home and industrial environments to military uses. Unlike the traditional computer networks, a WSN is application-oriented and deployed for a specific task. WSNs are data centric, which means that messages are not send to individual nodes but to geographical locations or regions based on the data content. A
WSN node is typically battery powered and characterized by extremely small size and low cost.

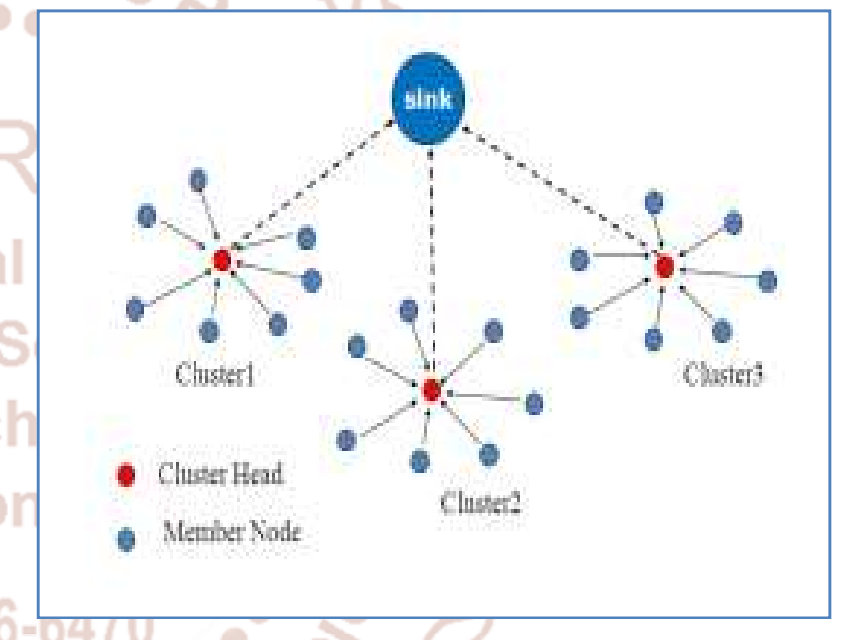

Fig. 1: Cluster based WSN

It is wireless network sometimes more specifically referred as Wireless Sensor and Actuator Networks as described in a Wireless sensor networks (WSNs) enable new applications and require non-conventional paradigms for protocol design due to several constraints. Wireless sensor network are using Global Positioning System (GPS) and local positioning algorithms can be used to positioning information and obtain location. The requirement for low device complexity together with low energy consumption, a proper balance between communication and signal/data processing capabilities must be found. 


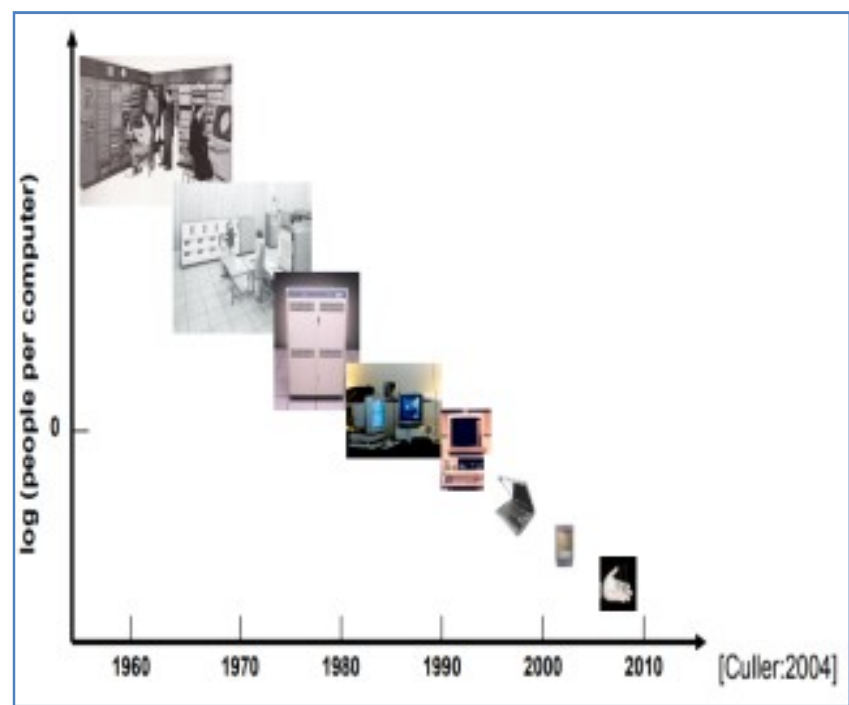

Fig. 2: WSN Technology

In recent years an efficient design of a Wireless Sensor Network has become a leading area of research. A Sensor is a device that responds and detects some type of input from both the physical or environmental conditions, such as pressure, heat, light, etc. The output of the sensor is generally an electrical signal that is transmitted to a controller for further processing.

\section{Types of WSNs (Wireless Sensor Networks)}

Depending on the environment, the types of networks are decided so that those can be deployed underwater, underground, on land, and so on. Different types of WSNs include:

1. Terrestrial WSNs

2. Underground WSNs

3. Underwater WSNs

4. Multimedia WSNs

5. Mobile WSNs

\section{ENERGY CONSUMPTION}

Wireless Sensor Network (WSN) plays an extremely significant role in usual lives. Wireless Networks in provisions of constraints of their resources. The energy consumption is the principal concern in Wireless Sensor Network (WSN). Therefore, a numerous researchers focused on energy efficient algorithms in WSNs for extending the life time of sensors. These differ depending on the deployment of node, the network design, the characteristics of the cluster head nodes and the network operation. Energy is proficient of save by grouping nodes as clusters.

\section{A. Cluster Head}

Clustering is used in order to advance the scalability of network performance. Clustering is useful in several sensor network applications such as inter cluster communication, node localization and so on. Clustering algorithms have extensive applications in the precedent years and common clustering algorithms have been proposed for energy consumption in recent years in all of these algorithms, and nodes are structured as clusters, superior energy nodes are called as Cluster Head $(\mathrm{CH})$ and other nodes are called as normal sensor nodes.

\section{LITERATURE REVIEW}

1. D. Naga Ravikiran and C. G. Dethe (2018) "Improvements in Routing Algorithms to Enhance Lifetime of Wireless Sensor Networks" International Journal of Computer Networks \& Communications (IJCNC) Vol.10 (2) 2018. In this paper improvements in various parameters are compared for three different routing algorithms. First, it is started with Low Energy Adaptive Cluster Hierarchy (LEACH)which is a famed clustering mechanism that elects a $\mathrm{CH}$ based on the probability model. Then, work describes a Fuzzy logic system initiated $\mathrm{CH}$ selection algorithm for LEACH.

2. Vineet Mishra et al. (2015). "Performance Analysis Of Cluster Formation In Wireless Sensor Networks", Global Journal of Advance Engineering Technologies and Sciences, 2(11). The energy consumption is the principal concern in WSN. It system is built on an IEEE 802.15.4 wireless mesh network. Wireless Sensor Networking is a network of wireless sensor nodes deployed in an area. The wireless sensor network consists of the sensor nodes. The idea of development of wireless sensor networks was initially motivated by military applications. A WSN provides a reliable, low maintenance, low power method for making measurements in applications where cabled sensors are impractical or otherwise undesirable. A wireless sensor network (WSN) is a wireless network using sensors to cooperatively monitor physical or environmental conditions such as humidity, pressure, temperature, sound, vibration.

3. T. Arivanantham et al. (2014). "Clustering Techniques to Analyze Communication Overhead in Wireless Sensor Network", International Journal of Computational Engineering Research, 4(5). The major problem with wireless sensor network is their limited source of energy, the courage constraint and high traffic load. In this paper we introduce various clustering techniques 
which are to be used to reduce communication overhead and increase network's lifetime. In the present work, the comparative evaluation of communication overhead for the wireless sensor network based on clustering technique is carried out. As a result of these experiments, we evaluated the communication overhead in WSN using Kmeans clustering algorithm and Fuzzy clustering algorithm.

4. Labisha R.V et al. (2014). "Energy Efficient Clustering Algorithms in Wireless Sensor Networks-An Analytical View" International journal 9(3). Wireless sensor network refers to a group of spatially distributed and dedicated sensors for monitoring and recording the physical conditions of environment like temperature, humidity, sound, pollution levels, and wind speed with direction and pressure. Sensors are self powered nodes which also possess limited processing capabilities and the nodes communicate wirelessly through a gateway. The capability of sensing, processing and communication found in sensor networks lead to a vast number of applications of wireless sensor networks in areas such as environmental monitoring, warfare, education, agriculture to name a few. In the present work, the comparative evaluation of communication overhead for the wireless sensor network based off on clustering technique is carried out.

5. Amrinder Kaur et al. (2013). "Simulation of Low Energy Adaptive Clustering Hierarchy Protocol for Wireless Sensor Network" International Journal of Scientific Engineering, 3(7). Recent advances in wireless sensor networks have settled into an important part of one's everyday life and gained attention from both the research community and actual users. In wireless sensor networks energy alertness is an essential consideration \& it explored to many new protocols specifically designed for sensor networks. This paper presents the overview of wireless sensor networks and their routing challenges and design issues that is faced in designing a protocol for sensor networks.

6. Anshul Shrotriya et al. (2013). "Energy Efficient Modeling of Wireless Sensor Networks Based on Different Modulation Schemes Using QualNet" International Journal of Scientific Engineering and Technology, 1(3). I have study in the paper that it account for the analysis of energy consumption in existing energy models of Wireless Sensor
Network (WSN) based upon different modulation. There are two main energy models preferred in Wireless Sensor Network, MOTES and MICAZ. So here, we have investigated the more energy efficient energy model under different modulation schemes. So I can simply find the better energy model as well as the better modulation scheme to efficiently utilize the energy in WSN. It has been found that, based upon the modulation schemes; mica $\mathrm{z}$ performs better than mica mote energy model.

7. Shiv Prasad Kori et al. (2013). "Performance Comparison in Terms of Communication Overhead for Wireless Sensor Network Based on Clustering Technique" International Journal of Electronics Communication and Computer Engineering, 4(3). The present work, the comparative evaluation of communication overhead for the wireless sensor network based of on clustering technique is carried out. It has been be observed that overhead in cluster based protocol is not much dependent upon update time. Simulation a result indicates that cluster based protocol has low communication overheads compared with the BBM based protocol when sink mobility is high. In these applications a large number of sensors are expected, requiring careful architecture and management of the network. Grouping nodes into clusters has been the most popular approach for support scalability in WSNs. Significant attention has been paid to clustering strategies and algorithms yielding a large number of publications.

\section{METHODOLOGY}

The System has been implemented in the MATLAB. The wireless sensor network is design with following specification in table 1 . The method of design simulation has been given below:

Table 1: Specification Fuzzy Algorithms

\begin{tabular}{|l|l|l|}
\hline S. No. & \multicolumn{1}{|c|}{ Specification } & \multicolumn{1}{|c|}{ Value } \\
\hline 1 & $\begin{array}{l}\text { NO. of Node and } \\
\text { cluster }\end{array}$ & $\begin{array}{l}200, \quad 300 \text { and } \\
400\end{array}$ \\
\hline 2 & $\begin{array}{l}\text { Length of network } \\
\text { area }\end{array}$ & $1 \times 1 \mathrm{~m}$ \\
\hline 3 & Maximum range & $500 \mathrm{~m}$ \\
\hline 4 & Noise power in $\mathrm{dBm}$ & $50 \mathrm{dbm}$ \\
\hline 5 & Transmitted power & $1 \mathrm{Mw}$ \\
\hline 6 & Operating frequency & $2.4 \mathrm{GHZ}$ \\
\hline 7 & Technology & Fuzzy \\
\hline
\end{tabular}




\section{A. Fuzzy C-Means Clustering}

This algorithm works by assigning membership to each data point corresponding to each cluster center on the basis of distance between the cluster center and the data point. For more the data is near to the cluster center more is its membership towards the particular cluster center. In clearly, summation of membership of each data point should be equal to one.

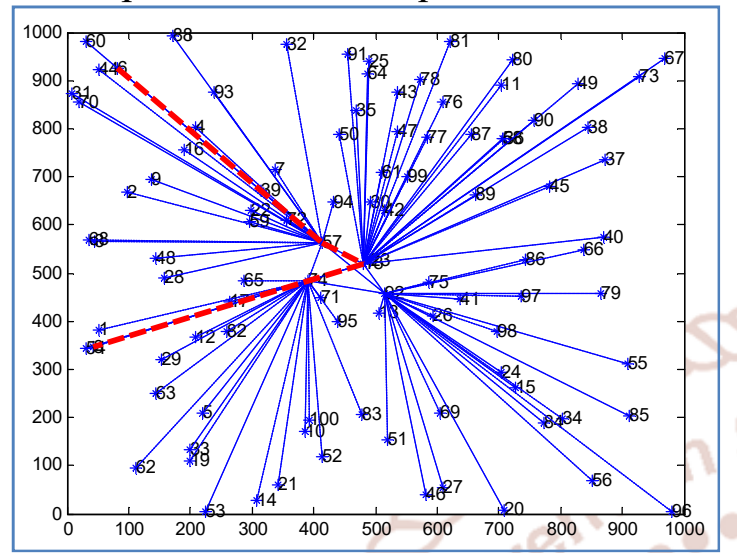

Fig: 3: Performance of Fuzzy clustering for 100 Nodes

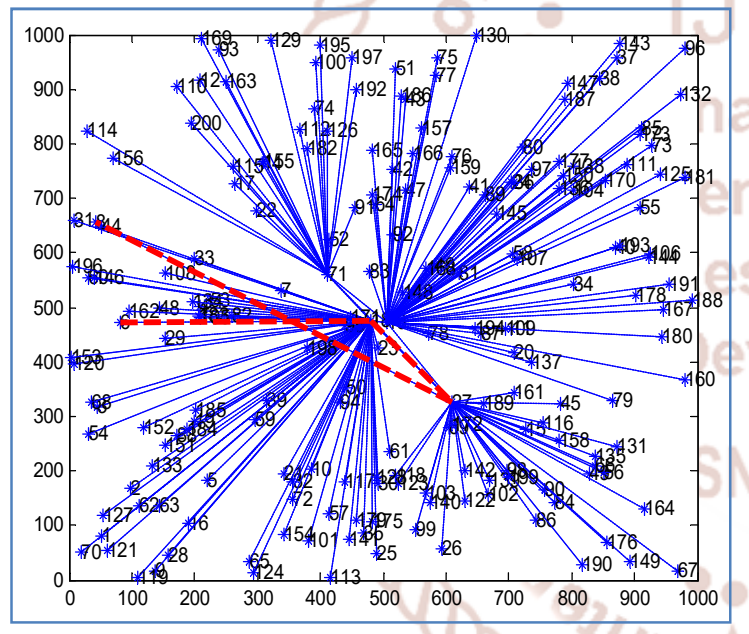

Fig: 4: Performance of Fuzzy clustering for 200 Nodes

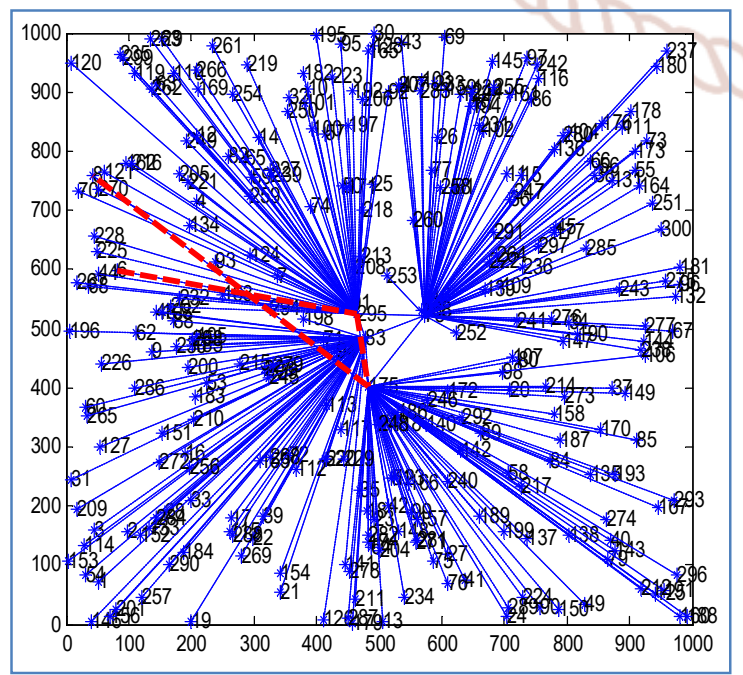

Fig: 5: Performance of Fuzzy clustering for 300 Nodes

\section{CONCLUSION}

Wireless network is considered as most common service used in industrial and commercial application due to its technological enhancement in the process, interaction and utilisation of low power embedded computational devices. Wireless sensor networks (WSNs) utilize fast, cheap, and effective applications to imitate the human intelligence capability of sensing on a wider distributed scale The energy consumption reduction has been taken as a objective in this project. The clustering based network architecture has been proposed in this project to reduce the communication overhead which in turn reduces the energy consumption. The various clustering techniques are available for locating the access point for WSN. Following path is generated for consider number of nodes 100, 200 and 300 respectively using Matlab toll R2013a.

Path $=6,57,18,8$ (Fig. 3)

Path $=6,18,27,8$ (Fig. 4)

Path $=6,295,175,8$ (Fig. 5)

\section{REFERENCES}

1. D. Naga Ravikiran and C.G. Dethe, "Improvements in Routing Algorithms to Enhance Lifetime ic of Wireless Sensor Networks" International Journal of Computer Networks \& Communications (IJCNC) Vol.10 (2) 2018.

2. Amrinder Kaur, "Simulation of Low Energy Adaptive Clustering Hierarchy Protocol for Wireless Sensor Network", Volume 3, Issue 7, July 2013.

3. Amitabh Basu, Jie Gao, Joseph S. B. Mitchell, Girishkumar Sabhnani , "Distributed localization using noisy distance and angle information" 7th AC international symposium on Mobile ad hoc networking and computing, 2006,pp. $262-273$.

4. Rui Zhang,Myung J.Lee, Seong-Soon Joo, "Mobile sink update local information through these Aps", 2008.

5. Shiv Prasad Kori, "Performance Comparison in Terms of Communication Overhead for Wireless Sensor Network Based on Clustering Technique", Volume 4, Issue 3, ISSN (Online): 2249-071X, ISSN (Print): 2278-4209.

6. Labisha R.V, 2Baburaj E, "Energy Efficient Clustering Algorithms in Wireless Sensor Networks-An Analytical View", Volume9, Number3, May 2014. 
7. Abdul Sattar Malik, Jingming Kuang, Jiakang Liu, Wang Chong, Performance Analysis of Clusterbased Wireless Sensor Networks with Application Constraints, I.J. Computer Network and Information Security, 2009, 1, 16-23

8. Performance evaluation of routing protocols for packet drop statistics for Meshed routing in IEEE 802.15.4 based WSNs

9. A. Allirani, and M. Suganthi, An Energy Efficient Cluster Formation Protocol with Low Latency In Wireless Sensor Networks, Vol:3 2009-03-21.

10. Eugene Shih, SeongHwan Cho, Nathan Ickes, Rex Min, Amit Sinha, Alice Wang, Anantha
Chandrakasan," Physical layer driven protocol and algorithm design for energy efficient wireless sensor networks", Proc. MOBICOM, 2001, pp. 272-287.

11. E. Ekici , S. Vural, J. McNair, D. Al-Abri "Secure probabilistic location verification in randomly deployed wireless sensor networks " Elsevier Science Publishers B. V,April 2006 ,pp.,195-209

12. E. Elnahrawy, X. Li, R. Martin, "The Limits of Localization using RSSI", in Proceedings of SECON, 2004.

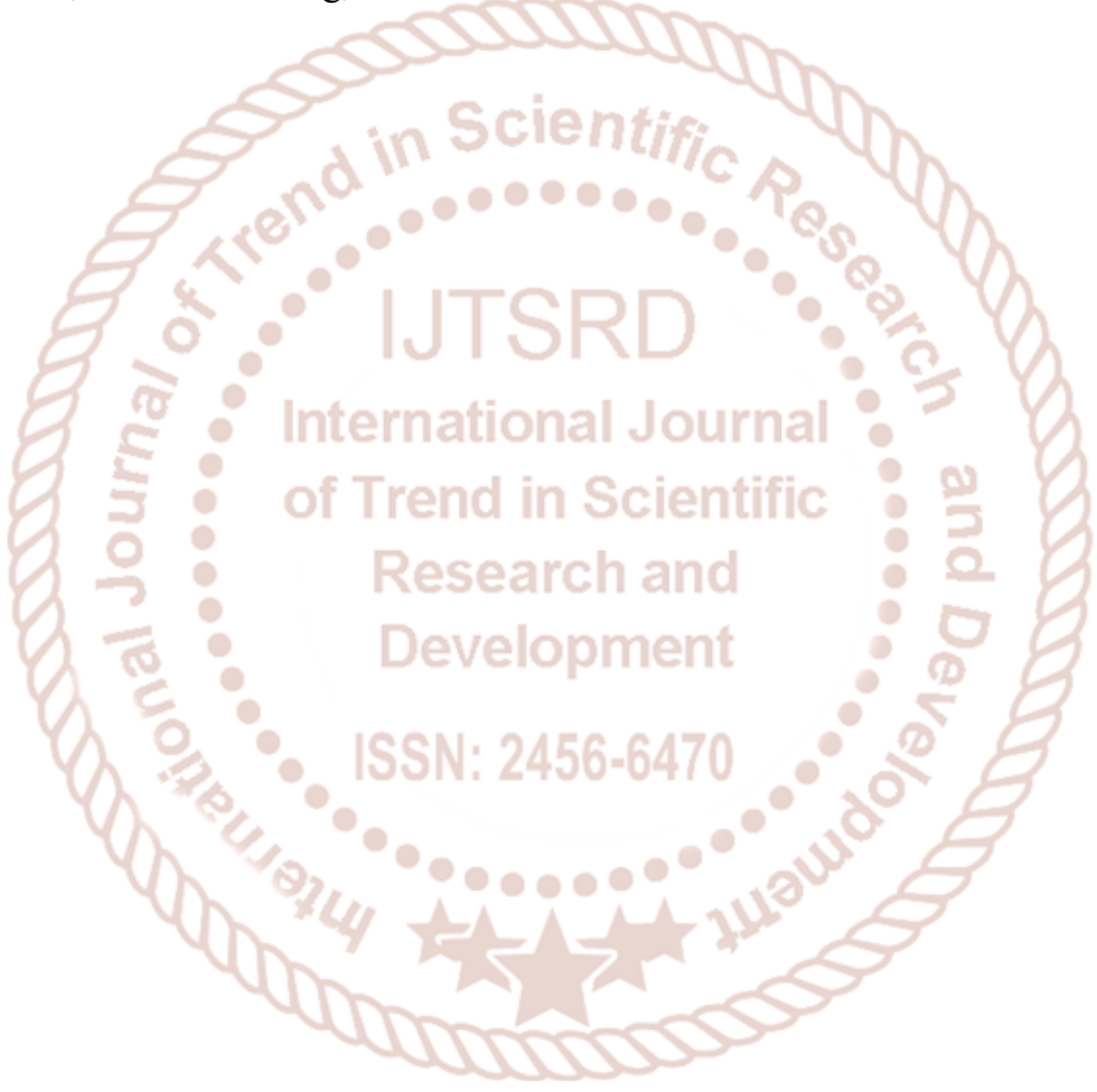

